\title{
Neonatal programming by immunological challenge: effects on ovarian function in the adult rat
}

\author{
Xue-Qing $\mathrm{Wu}^{1,3}$, Xiao-Feng $\mathrm{Li}^{1}$, Bilu Ye ${ }^{3}$, Neha Popat ${ }^{1}$, Stuart R Milligan ${ }^{1}$, Stafford L Lightman ${ }^{2}$ \\ and Kevin T O'Byrne ${ }^{1,3}$ \\ ${ }^{1}$ Division of Women's Health, School of Medicine, King's College London, Guy's Campus, 2.95W, Hodgkin Building, \\ London SE1 1UL, UK, ${ }^{2}$ Henry Wellcome Laboratory for Integrative Neuroscience and Endocrinology, University of \\ Bristol, Bristol BS1 3NY, UK and ${ }^{3}$ First Affiliated Hospital of Wenzhou Medical College, Wenzhou 325000, People's \\ Republic of China
}

Correspondence should be addressed to K T O'Byrne at Division of Women's Health, School of Medicine, King's College London; Email: kevin.o'byrne@kcl.ac.uk

\begin{abstract}
Neonatal exposure to an immunological challenge (lipopolysaccharide, LPS) increases the activity of hypothalamo-pituitary-adrenal axis and sensitises the GNRH pulse generator to the inhibitory influence of stress in adult rats. We investigated the effects of neonatal exposure to LPS on various reproductive parameters during puberty and into adulthood in female rats. LPS $(50 \mu \mathrm{g} / \mathrm{kg}$, i.p.) or saline was administered on postnatal days 3 and 5. Vaginal opening was recorded, and oestrous cyclicity was monitored immediately post puberty and again at 8-9 weeks of age. At 10 weeks of age, the ovaries were removed and the number of follicles was counted, together with the thickness of the theca interna of the largest antral follicles. Ovarian sympathetic nerve activity was assessed immunohistochemically by measurement of the levels of ovarian low-affinity receptor of nerve growth factor (p75NGFR). In rats exposed to LPS in early life, there was a significant delay in puberty and disruption of oestrous cyclicity immediately post puberty, which persisted into adulthood. The follicle reserve was decreased, the thickness of the theca interna increased and the expression profile of ovarian p75NGFR increased in the neonatal LPS-treated animals. These data suggest that exposure to LPS during early neonatal life can have long-term dysfunctional effects on the female reproductive system, which might involve, at least in part, increased ovarian sympathetic nerve activity.

Reproduction (2011) 141 241-248
\end{abstract}

\section{Introduction}

It is well known that stressful stimuli can have profound suppressive effects on the activity of the hypothalamopituitary-gonadal axis, especially the GNRH pulse generator, the central regulator of reproduction (Ferin 2006). We have previously shown that neonatal exposure to lipopolysaccharide (LPS) as an immunological challenge programmes enhanced stress responsiveness of the hypothalamo-pituitary-adrenal (HPA) axis in adult rats, with increases in basal corticosterone pulse frequency and amplitude, as well as marked increases in stress-induced corticosterone release (Shanks et al. 1995, 2000). These changes are due, in part, to decrease in hippocampal and hypothalamic glucocorticoid receptors, which attenuate corticosterone negative feedback (Shanks et al. 1995, 2000). We have recently shown that exposure to LPS in early neonatal life results in long-term sensitisation of the GNRH pulse generator to the inhibitory influence of stress in adulthood (Li et al. 2007). Perinatal endotoxin exposure has also been shown to decrease circulating levels of progesterone and to increase testosterone in adult female rats (Nillson et al. 2001). Endotoxin, or interleukin 1, a key mediator of endotoxin effects, has been found to disrupt ovarian cyclicity in the adult of various species including the rat, cow, sheep and monkey (Peter et al. 1989, Rivest et al. 1993, Xiao et al. 1998, Battaglia et al. 2000). We have recently shown that the critical period for a significant delay in puberty (as defined by vaginal opening and first vaginal oestrus) as a result of neonatal LPS exposure is before 7 days of age (Knox et al. 2009), but there have been no reported studies on whether ovarian cyclicity is affected or whether reproductive effects persist into later life.

In addition to activation of the HPA axis, stressors activate the sympathetic nervous system (Zhou \& Jones 1993, Xia \& Krukoff 2003). Central administration of endotoxin has been demonstrated to activate the catecholaminergic system in the hypothalamus and 
brainstem, including increasing tyrosine hydroxylase $(T H)$ mRNA levels in locus coeruleus, the central noradrenergic system of the brain (Xia \& Krukoff 2003). Furthermore, endotoxin challenge increases circulating levels of noradrenaline and adrenaline in the rat (Zhou \& Jones 1993). Stress-induced increase in systemic sympathetic activity can also increase sympathetic tone in the ovary. Chronic intermittent cold or restraint stressinduced increase in sympathetic nerve activity is associated with increased noradrenaline, nerve growth factor (NGF) and its receptor (p75NGFR) in the ovary (Paredes et al. 1998, Dorfman et al. 2003). Furthermore, the well-established polycystic ovary (PCO) rat model induced by oestradiol valerate administration, which is associated with increased ovarian sympathetic tone, shown by an overproduction of noradrenaline (Lara et al. 1993), NGF and p75NGFR in the ovary (Lara et al. 2000), is accompanied by irregular oestrous cycles and infertility (Lara et al. 2000). Neonatal exposure to oestradiol valerate similarly increases ovarian sympathetic activity resulting in ovarian dysfunction in adult rats (Sotomayor-Zarate et al. 2008).

The aim of this study is to test the hypothesis that exposure to the bacterial endotoxin, LPS, in early neonatal life, which delays puberty and enhances stress responsiveness, is associated with ovarian dysfunction in adulthood, as manifested by alterations in ovarian cyclicity and morphology and expression of ovarian p75NGF, a marker of sympathetic nerve activity.

\section{Results}

\section{Neonatal LPS exposure delayed vaginal opening and first vaginal oestrus}

Neonatal exposure to LPS resulted in a significant delay in both the day of vaginal opening (neo-LPS: $40.3 \pm 0.8$; neo-saline: $38.2 \pm 0.5 ; P<0.05)$ and the day of first vaginal oestrus (neo-LPS: $41.2 \pm 0.7$; neo-saline: 38.6 $\pm 0.6 ; P<0.05)$. However, there was no significant difference in body weight gain throughout the experimental period between neonatal LPS- and neonatal saline-treated groups (Fig. 1).

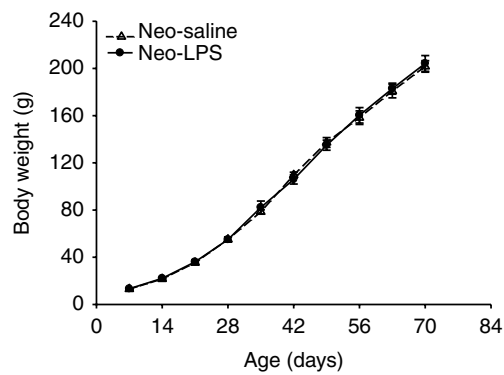

Figure 1 Effects of neonatal exposure to LPS (LPS, $50 \mu \mathrm{g} / \mathrm{kg}$ i.p.) on postnatal day 3 and 5 on body weight. There was no significant difference in body weight gain between neonatal LPS (neo-LPS)-treated and neonatal saline (neo-saline) controls ( $n=37-42$ per group).
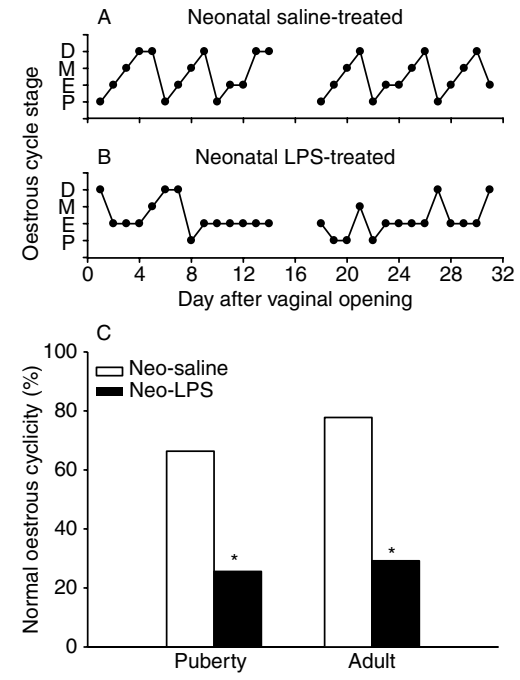

Figure 2 Effects of neonatal exposure to LPS on oestrous cyclicity immediately post puberty and in adulthood. Representative examples of oestrous cyclicity are illustrated in (A) and (B). Normal oestrous cyclicity is defined as having at least two consecutive normal cycles (i.e. cycle length 4-5 days; oestrus phase 1-2 days). The percentage of normal oestrous cyclicity was decreased at puberty and in adulthood of neonatal LPS-treated rats compared with neonatal saline controls $(\mathrm{C})$. $P$, prooestrus; $E$, oestrus; $M$, metoestrus; $D$, dioestrus. ${ }^{*} P<0.05$ versus neonatal saline controls ( $n=37-42$ per group).

\section{Neonatal LPS exposure disrupted oestrous cyclicity immediately post puberty and in adulthood}

Representative examples of oestrous cycles in each treatment group are illustrated in Fig. 2A and B. The majority $(66.4 \%)$ of the neonatal saline-treated rats showed normal, 4-5 days oestrous cycles immediately post puberty, and the percentage of normal cyclicity increased to $77.8 \%$ in adulthood (Fig. 2C). In neonatal LPS-treated rats, only $26.2 \%$ showed normal cyclicity immediately post puberty, and this disruption persisted into adulthood, with only $29.2 \%$ of them showing normal cyclicity (Fig. 2C). The percentage of normal oestrous cycles was significantly lower in neonatal LPS-treated rats, both immediately post puberty and in adulthood compared with saline controls (Fig. 2C; $P<0.05$ ).

\section{Effects of neonatal LPS exposure on ovarian weight and morphology in adulthood}

The mean \pm S.E.M. of ovarian weight at 10 weeks of age for the neonatal saline- and neonatal LPS-treated groups was not significantly different $(70.3 \pm 3.1$ and 72.9 $\pm 3.2 \mathrm{mg}$ respectively). There was a significant decrease in the total number of primordial, primary, preantral and antral follicles in neonatal LPS-treated females, compared with neonatal saline-treated control animals (Table 1). In addition, the thickness of the theca interna layer in the largest antral follicle was significantly 
Table 1 Number of different types of follicles in the ovaries of adult rats treated neonatally (postnatal day $3+5$ ) with saline (neo-saline) or lipopolysaccharide (neo-LPS).

\begin{tabular}{lccc}
\hline Follicle type & Neo-saline $(n=4)$ & Neo-LPS $(n=4)$ & $P$ value \\
\hline Primordial & $355.0 \pm 7.3(344-369)$ & $210.0 \pm 18.8(176-241)^{*}$ & 0.002 \\
Primary & $431.0 \pm 20.1(394-463)$ & $254.3 \pm 41.1(172-297)^{*}$ & 0.018 \\
Preantral & $63.3 \pm 5.8(56-75)$ & $39.0 \pm 6.2(27-48)^{*}$ & 0.047 \\
Antral & $28.6 \pm 1.7(26-32)$ & $14.0 \pm 2.8(9-14)^{*}$ & 0.012 \\
\hline
\end{tabular}

Values are mean \pm S.E.M. (range). ${ }^{*} P<0.05$ versus neonatal saline controls (Student's $t$-test).

increased at oestrus, metoestrus and dioestrus in the ovaries from neonatal LPS-treated rats compared with neonatal saline controls (Fig. 3).

\section{Effects of neonatal LPS exposure on sympathetic tone in the adult ovary}

The immunohistochemical staining for p75NGFR was generally confined to the theca cells, especially the theca interna of growing follicles (preantral and antral follicles; Fig. 4A-D). Neither primordial nor primary follicles had specific staining for p75NGFR. There was no obvious specific staining for p75NGFR in the granulosa cells and corpora lutea, but there was some staining in the interstitial tissue. There was no significant difference in staining intensity between the different phases of the oestrous cycle in the neonatal LPS-treated rats or neonatal saline controls (Fig. 4E and F). However, the intensity of staining for p75NGFR in the theca interna of preantral (Fig. 4E) and antral (Fig. 4F) follicles was significantly enhanced in neonatal LPS-treated rats compared with neonatal saline controls $(P<0.05)$.

\section{Discussion}

This study demonstrates that neonatal exposure to LPS in female rats can have long-term effects on reproductive function. Not only were markers of puberty onset (vaginal opening and first vaginal oestrus) delayed (in agreement with the findings of Knox et al. (2009)), but in addition, oestrous cyclicity was disrupted both immediately post puberty and into adulthood. The disruption to oestrous cyclicity was characterised by a persistent or prolongation of vaginal oestrus. Although the mechanisms underlying this disruption remain to be established, it has been reported that corticosterone administered on postnatal days (pnd) 3 and 6 induced disruption of oestrous cycles immediately post puberty and in adulthood, which again was characterised by persistent vaginal oestrus (Turner \& Taylor 1977). It has been shown that increased levels of glucocorticoid can also block LH surges or ovulation in rats (Baldwin 1979, Roozendaal et al. 1997) and sheep (Macfarlane et al. 2000). Hypercortisolism is associated with functional hypothalamic amenorrhoea in women (Suh et al. 1988,
Brundu et al. 2006). Since we have previously shown that adult rats treated neonatally with LPS (using an identical protocol to this study) are known to exhibit hypercorticosteronaemia (Shanks et al. 2000), it is possible that this may underlie the disruption of oestrous cyclicity observed in this study. We have recently shown that there is a critical time window for neonatal LPS treatment to delay puberty in the rat, with treatment delayed to pnd 7 and 9 or pnd 14 and 16 no longer effective (Knox et al. 2009), consistent with the observation that oestrous cyclicity was unaffected in adult rats administered with LPS on pnd 10 (Iwasa et al. 2009). It is possible that a similar critical time window may operate for long-term programming effect of an immunological challenge on oestrous cyclicity.
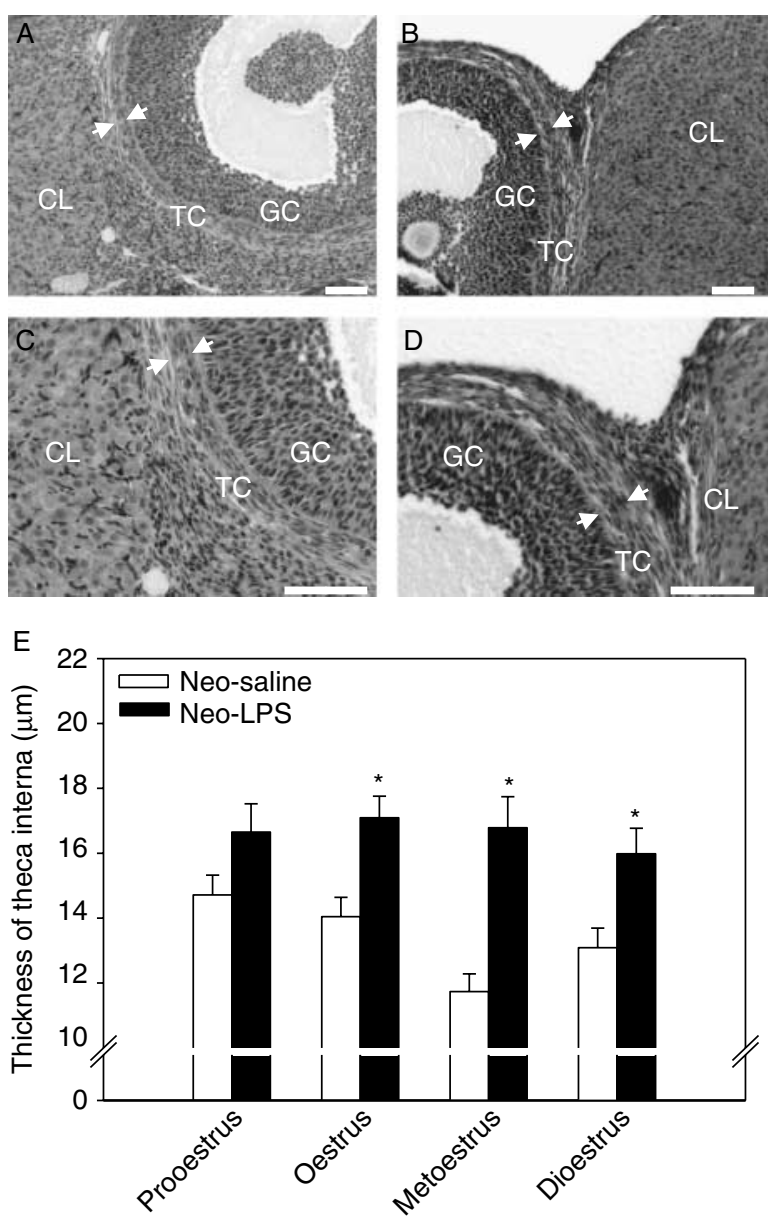

Figure 3 Effects of neonatal exposure to LPS on the thickness of the theca interna layer of the largest follicles in adult ovary. Representative examples of the theca interna layer of the largest follicle in adult ovaries are illustrated in A and C (neonatal saline controls) and B and D (neonatal LPS-treated rats). The thickness of the theca interna layer of the largest follicles was increased at oestrus, metoestrus and dioestrus in neonatal LPS-treated rats compared with neonatal saline controls (E). $\mathrm{CL}$, corpus luteum; GC, granulosa cell; TC, theca cell; arrows show the theca interna layer. Photomicrographs illustrate dioestrus stage of oestrous cycle. Scale bar $=50 \mu \mathrm{m}$. ${ }^{*} P<0.05$, versus neonatal saline controls ( $n=8-11$ for each group). 

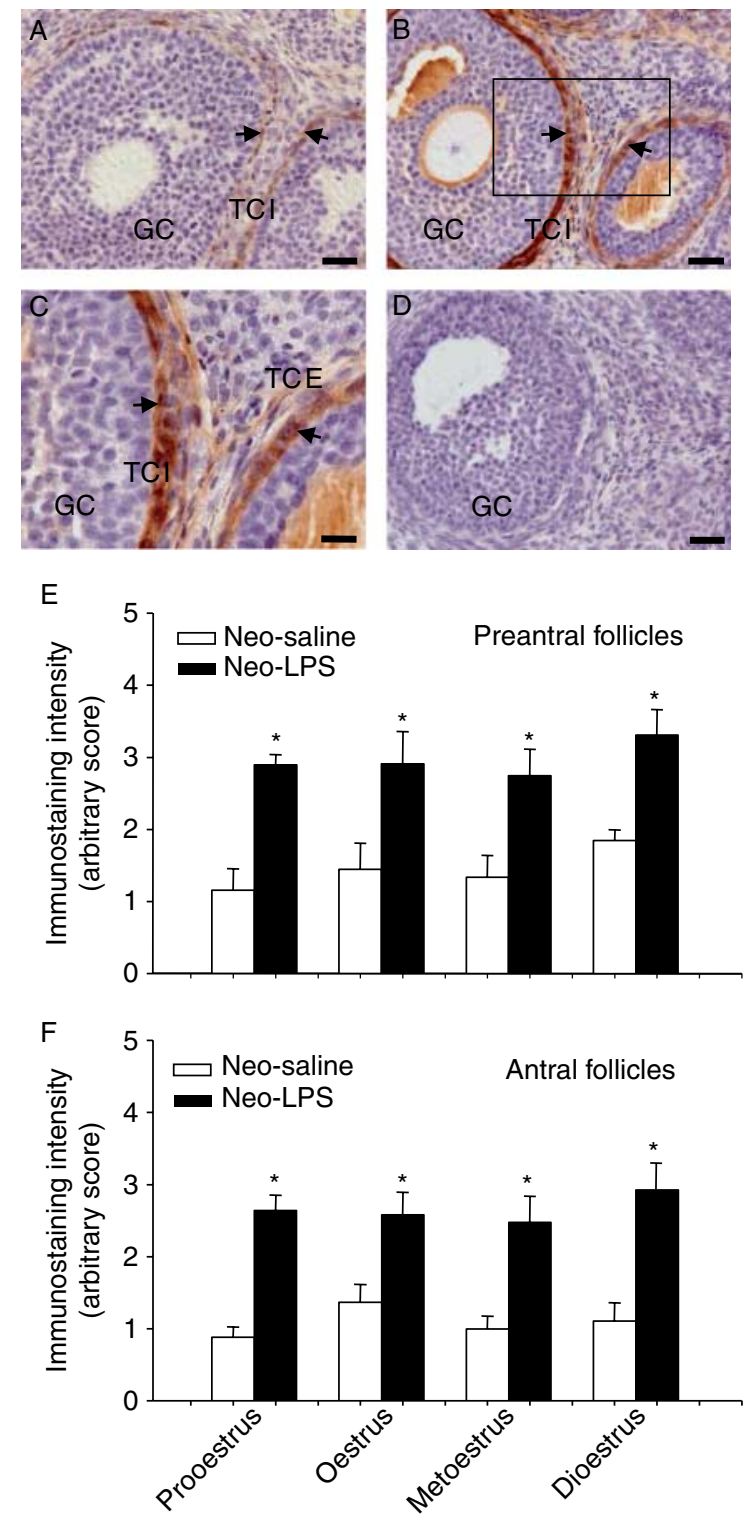

Figure 4 Effects of neonatal exposure to LPS on the immunoreactivity of p75NGFR in theca interna cells of the adult ovary. Low intensity of immunoreactivity for p75NGFR was detected in theca cells, especially theca interna (arrows) of growing follicles (preantral and antral follicles) from neonatal saline controls (A). The immunostaining for p75NGFR in the theca interna cells of preantral and antral follicles was significantly higher in the neonatal LPS-treated rats (B). (C) shows a higher magnification of the boxed area in (B). (D) is a negative control with no staining in the adjacent section incubated in absence of primary antibody. $\mathrm{H}$-scores of immunostaining intensity for p75NGFR in theca interna cells are summarised in (E) and (F). There was no significant difference in staining intensity between different phases of the oestrous cycle. GC, granulosa cell; $\mathrm{TCl}$, theca interna cell; TCE, theca externa cell. Photomicrographs illustrate dioestrus stage of oestrous cycle. Scale bars: A, B and D=20 $\mu \mathrm{m} ; \mathrm{C}=10 \mu \mathrm{m} .{ }^{*} P<0.05$, versus neonatal saline controls ( $n=8-11$ for each group).
In contrast to our findings, Nillson et al. (2002) failed to show an effect of neonatal LPS treatment on pnd 3 and 5 on oestrous cyclicity in adult rats. The reason for this discrepancy is unknown, although different strains of rat and different routes of LPS were used.

The neuropeptide kisspeptin (KISS1) and its receptor (KISS1R) have been identified as an essential part of the hypothalamic circuits that govern the initiation of puberty (Navarro et al. 2007). Additionally, KISS1/KISS1R signalling plays a critical role in controlling normal ovarian cyclicity in the adult through its regulation of the GNRH pulse generator ( $\mathrm{Li}$ et al. 2009) and the GNRH surge generator (Kinoshita et al. 2005, Pineda et al. 2010). We found that neonatal LPS administration caused a significant decrease in Kiss1 mRNA expression and increase in Kiss1r mRNA expression in medial preoptic area (mPOA) in adult animals (Knox et al. 2009). A similar change in the Kiss1/Kiss1r expression profile was observed in the $\mathrm{mPOA}$ in response to both acute or chronic physiological stress levels of corticosterone (Kinsey-Jones et al. 2009). Thus, the altered hypothalamic Kiss1/Kiss1r expression by neonatal exposure to LPS may account for, at least in part, the disruption of oestrous cyclicity observed in this study.

It is well established that normal ovarian cyclicity and ovulation rely on both normal pulsatile and preovulatory surge release of FSH and LH. Increased levels of corticosterone have been shown to decrease levels of FSH in rats (Valli et al. 2000, Baravalle et al. 2007). It is possible that the increased basal levels of corticosterone in adult rats treated neonatally with LPS (Shanks et al. 2000) may decrease FSH secretion, leading to the decreased number of preantral and antral follicles observed in this study. We have previously shown that neonatal LPS treatment sensitises the GNRH pulse generator to the inhibitory effect of stress in adulthood (Li et al. 2007), although basal pulsatile LH secretion was not affected. However, in this study we used gonadalintact rats instead of ovariectomised, oestradiol-replaced animals as in our previous study. Other studies have already shown that increased corticosterone levels in ovary-intact rats were accompanied by a reduction in $\mathrm{LH}$ secretion (Valli et al. 2000, Baravalle et al. 2007). Additionally, elevated levels of glucocorticoid or restraint stress have been shown to delay, attenuate or block the preovulatory LH surge in sheep (Wagenmaker et al. 2009) and rats (Baldwin 1979, Roozendaal et al. 1997). Whether the LH surge is also affected by the administration of LPS in early life needs to be investigated further.

The total number of follicles, in particular primordial follicles, was decreased in adult rats treated neonatally with LPS. This suggests that early life exposure to an immunological challenge may affect the earliest stages of folliculogenesis, when primordial follicles are organising. It has been demonstrated that follicles are assembled and differentiated after pnd 2 in rats (Rajah et al. 1992, 
Kezele et al. 2002), so the administration of LPS on pnd 3 and 5 may coincide with the critical developmental time of primordial follicles assembly and transition. Therefore, we speculate that neonatal exposure to LPS reduced the reserve of ovarian follicles and impaired fertility later in life by interfering with the assembly of primordial follicles and, consequently, transition from primordial to secondary follicles. Indeed, a reduced follicular pool size has been shown to result in an increased incidence of irregular cyclicity (Anzalone et al. 2001).

Whether the increase in p75NGFR, a marker of ovarian sympathetic nerve activity (Lara et al. 2000), observed in this study, is due to the up-regulation of central sympathetic activity or local peripheral regulatory mechanism and its role in controlling ovarian function need further studies. The thickness of the theca interna layer of the largest follicle in adult ovaries was increased by neonatal LPS treatment. This may be due to increased ovarian sympathetic tone since previous studies using chronic intermittent cold stress, which classically enhances ovarian sympathetic nerve activity, as measured by increased noradrenaline and NGF in the ovary, are similarly accompanied by increased thickness of the theca layer in the rat ovary (Dorfman et al. 2003). Interestingly, it was recently shown that exercise or acupuncture reversed the increased thickness of the theca interna in the dihydrotestosterone-induced PCOS rat ovary (Mannerås et al. 2009). In this study, the immunoreactivity for p75NGFR, the common receptor for neurotrophins, which are necessary for growth and differentiation of ovarian sympathetic nerves (Dissen et al. 2002), was increased in ovaries from neonatal LPS-treated rats. These data suggest that ovarian sympathetic activity may have been increased by neonatal exposure to LPS. The ovarian sympathetic innervation has been shown to participate in the control of ovarian function such as follicular development, steroid secretion and ovulation (Ojeda \& Lara 1989, Lara et al. 1990). However, increased sympathetic activity in the ovary has been shown to derange follicular development, disrupt oestrous cyclicity and reduce ovulation in stress animal models (Paredes et al. 1998, Dorfman et al. 2009) and oestradiol valerate-induced PCO rats (Rosa-E-Silva et al. 2003, Greiner et al. 2005). Transgenic mice with increased NGF level in the ovary show delay in puberty and disruption of oestrous cyclicity (Dissen et al. 2009). Furthermore, immunoneutralising NGF action in conjunction with blocking the synthesis of p75NGFR restored oestrous cyclicity and ovulatory capacity in oestradiol valerate-treated rats (Lara et al. 2000). Moreover, there is an increased density of ovarian catecholaminergic nerves (Heider et al. 2001) and increased sympathetic nerve activity measured by direct intraneural recordings in PCOS patients (Sverrisdöttir et al. 2008). The increase in p75NGFR, a key marker of sympathetic activity observed in the ovary in this study, may therefore account for the derangement of follicular development, increased thickness of the theca interna and disruption of oestrous cyclicity.

Several stressors, including LPS, have been shown to increase sympathetic nerve activity in adult rats (Zhou \& Jones 1993, Xia \& Krukoff 2003). It has been shown that the autonomic nervous systems play an important role in the regulation of ovarian physiology (Agudo 2002, Dissen et al. 2002). Viral transneuronal tracing studies have provided morphological evidence for direct innervation of the ovary via multisynaptic neuronal pathways, including the noradrenergic nucleus, the locus coeruleus (Gerendai et al. 1998). Cold stress, which promotes PCO through up-regulation of ovarian sympathetic activity in rats (Dorfman et al. 2003, 2009), is also accompanied by an increased activation of $\mathrm{TH}$-immunoreactive neurones in the locus coeruleus (Bernuci et al. 2008, Anselmo-Franci et al. 2009). Moreover, lesions in the locus coeruleus can reduce the increased ovarian noradrenergic activity, decrease the thickened theca interna layer and restore oestrous cycles (Bernuci et al. 2008).

In conclusion, this study shows that neonatal exposure to LPS leads to long-term dysfunction of the reproductive system at the ovarian level in rats, especially disruption of oestrous cyclicity and thickening on the theca interna, which might be associated with the increased ovarian sympathetic tone. Therefore, in addition to increasing the activity of the HPA axis (Shanks et al. 2000) and sensitising the hypothalamic GNRH pulse generator to the inhibitory effects of stress in adulthood ( $\mathrm{Li}$ et al. 2007), immunological challenge in early life can have a long-lasting suppressive effect on ovarian function.

\section{Materials and Methods \\ Animals and neonatal endotoxin exposure}

Pregnant Sprague-Dawley rats (Charles River, Margate, UK) were housed under controlled conditions $(12 \mathrm{~h}$ light: $12 \mathrm{~h}$ darkness cycle, with lights on at $0700 \mathrm{~h}$; temperature, 22 $\pm 2{ }^{\circ} \mathrm{C}$ ) and supplied with ad libitum food and water. On the day of birth (pnd 0), litters were culled to 10-12 pups. On pnd 3 and 5 , pups were injected i.p. with $50 \mu \mathrm{g} / \mathrm{kg}$ endotoxin in $0.05 \mathrm{ml}$ sterile saline (LPS, serotype Escherichia coli 055:B5; Sigma-Aldrich), a dose known to provoke permanent HPA axis activation in adulthood (Shanks et al. 1995, 2000) but to cause no discernable change in the general health of the pups or maternal behaviour (Shanks et al. 2000). Control pups received saline $(0.05 \mathrm{ml})$. Animals were randomly assigned to LPS or vehicle in the same litter. All litters were weaned at pnd 21, and female offspring were housed four to six per cage until they reached 10 weeks of age. All animal procedures were conducted under the British Home Office Animal Scientific Procedure Act 1986 (Project Licence 70/6237) and in accordance with accepted standards of the local ethical review committee. 


\section{Puberty onset and oestrous cyclicity monitoring}

Animals were monitored daily for vaginal opening from pnd 28. Once vaginal opening occurred, vaginal smears were taken and monitored daily for 2 consecutive weeks and again at 8-9 weeks of the age. The different stages of the oestrous cycle were determined according to the predominant cell type present in the vaginal smears under light microscope (Marcondes et al. 2002). Normal oestrous cyclicity was defined as having at least two consecutive normal oestrous cycles, which lasts for 4-5 days with 1-2 days of oestrus. The cycle length was determined by the number of consecutive days from the last day of a cornified smear to the last day of an oestrus smear in the subsequent cycle. Animals were weighed weekly until the end of the experiment.

\section{Ovarian morphology}

At 10 weeks of age, rats were ovariectomised. The right ovary was cleaned of fat tissue, weighed and fixed in $10 \%$ formaldehyde buffer for $20 \mathrm{~h}$ at room temperature. Waxembedded ovaries were sectioned longitudinally at $4 \mu \mathrm{m}$; every tenth section (six sections from each ovary) was mounted on a glass slide and stained with haematoxylin-eosin. The thickness of the theca interna layer of the largest follicle was determined with a calibrated scale bar in the microscope. For counting the total number of different types of follicles, the ovaries from a separate group of animals (neonatal LPS- or neonatal saline-treated rats) were completely sectioned at a thickness of $8 \mu \mathrm{m}$ per section, and each was subjected to analysis. Only follicles in which the nucleus of the oocyte was visible were counted (Lara et al. 1990). The sections were examined under a light microscope (Zeiss Axioskop 2 plus, Oberkochen, Germany) with an image analysis system (Axiovision 2.05; Zeiss) by two independent investigators blind to the treatment group.

The follicles were classified as following: primordial follicles with oocytes surrounded by one layer of flattened pregranulosa cells; primary - follicles with oocytes surrounded by no more than two layers of cuboidal granulosa cells; preantral - follicles without any antral cavity and with two or more layers of granulosa cells; antral - follicles with apparent cavity (Lara et al. 1990, 2000).

\section{Immunohistochemistry}

Immunohistochemical staining for p75NGFR was performed on $4 \mu \mathrm{m}$ paraffin embedded sections from ovaries of 10 -weekold rats. The sections were deparaffinised in xylene, hydrated in descending concentration of ethanol and incubated with $0.3 \%$ Triton X-100 (Sigma-Aldrich) in PBS to increase permeability before incubated with $0.3 \% \mathrm{H}_{2} \mathrm{O}_{2}$ to inhibit endogenous peroxidases and with non-immune goat serum (Vector Laboratories, Burlingame, CA, USA) in PBS to reduce background staining. Thereafter, the sections were incubated in 1:1000 monoclonal mouse anti-p75NGFR primary antibody (Chemicon International, Temecula, CA, USA) containing 2\% normal goat serum at $4{ }^{\circ} \mathrm{C}$ for $24 \mathrm{~h}$. The sections were then rinsed before incubation in 1:150 biotinylated goat anti-mouse
IgG (Vector Laboratories) for $90 \mathrm{~min}$ at room temperature followed by 1:200 conjugated avidin-biotin complex (Vector Laboratories) for an additional $45 \mathrm{~min}$ at room temperature. Visualisation of p75NGFR immunoreactivity was achieved using the $3^{\prime}, 3^{\prime}$-diaminobenzidine (Sigma-Aldrich). The sections were finally counterstained with $10 \%$ haematoxylin and coverslipped with DPX. Negative controls were run by incubating adjacent sections with non-immune serum and omission of the primary antibody. The sections from control and LPS-treated animals were run together in the same batch. Immunohistochemical detection of rat spinal cord was included as a positive control for p75NGFR in each batch of experiments. In each immunohistochemical experiment, two control sections from the same ovary tissue were included as immunostaining quality controls for the variation between different batches of experiments.

Semi-quantitative analyses of immunostaining intensity for p75NGFR were carried out on a Zeiss AxioVision microscope image system (Zeiss). All analyses were performed on coded slides by two independent investigators blind to the treatment groups.

\section{Statistical analyses}

Comparisons between neonatal LPS and neonatal saline treatment groups on vaginal opening, first oestrus and body weight were made by subjecting data to one-way ANOVA followed by Dunnett's test. The percentage of normal oestrous cycles between groups was compared using $\chi^{2}$ test. Comparisons between groups on thickness of the theca interna layer and follicle number were made by subjecting data to the MannWhitney $U$ test and Student's $t$-test respectively. All data are shown as mean \pm S.E.M.

The intensity of immunostaining for p75NGFR was assessed by using a modification of a semi-quantitative $H$-score method described by Akercan et al. (2008). Semi-quantitative immunohistochemical $H$-score values were calculated from the intensity and percentage of cells staining at each intensity. Intensities were classified as 0 (no staining), +1 (weak staining), +2 (weak-moderate staining), +3 (moderate staining), +4 (strong staining) and +5 (very strong staining). For each slide, the $H$-score value was calculated using the equation: $\Sigma(I \times P C)$, where $I$ and $P C$ represent intensity and percentage of cells that stain at each intensity respectively. Data were expressed as mean \pm s.E.M. The different groups were analysed by the Mann-Whitney $U$ test. $P$ values $<0.05$ were considered statistically significant.

\section{Declaration of interest}

The authors declare that there is no conflict of interest that could be perceived as prejudicing the impartiality of the research reported.

\section{Funding}

This work was supported by Wellcome Trust and BBSRC. $\mathrm{X}-\mathrm{Q}$ Wu sponsored by a KC Wong PhD studentship. 


\section{References}

Agudo LI 2002 Role of the central and peripheral nervous system in the ovarian function. Microscopy Research and Technique 59 462-473. (doi:10.1002/jemt.10232)

Akercan F, Cirpan T, Terek C, Ozcakir HT, Giray G, Sagol S \& Karadadas N 2008 The immunohistochemical evaluation of VEGF in placenta biopsies of pregnancies complicated by preeclampsia. Archives of Gynecology and Obstetrics 277 109-114. (doi:10.1007/s00404-007-0430-5)

Anselmo-Franci JA, Bernuci MP, Leite CM, Leoni GB \& Szawka RE 2009 Central and peripheral noradrenergic activities during cold stressinduced polycystic ovary in rats. Program of the 39th Annual Meeting of the Society for Neuroscience, Chicago, USA. Abstract 868.1.

Anzalone CR, Hong LS, Lu JK \& LaPolt PS 2001 Influence of age and ovarian follicular reserve on estrous cycle patterns, ovulation, and hormone secretion in the Long-Evans rat. Biology of Reproduction 64 1056-1062. (doi:10.1095/biolreprod64.4.1056)

Baldwin DM 1979 The effect of glucocorticoids on estrogen-dependent luteinizing hormone release in the ovariectomized rat and on gonadotropin secretion in the intact female rat. Endocrinology 105 120-128. (doi:10.1210/endo-105-1-120)

Baravalle C, Salvetti NR, Mira GA, Lorente JA \& Ortega HH 2007 The role of ACTH in the pathogenesis of polycystic ovarian syndrome in rats: hormonal profiles and ovarian morphology. Physiological Research 56 67-78.

Battaglia DF, Krasa HB, Padmanabhan V, Viguie C \& Karsch FJ 2000 Endocrine alterations that underlie endotoxin-induced disruption of the follicular phase in ewes. Biology of Reproduction 62 45-53. (doi:10. 1095/biolreprod62.1.45)

Bernuci MP, Szawka RE, Helena CV, Leite CM, Lara HE \& AnselmoFranci JA 2008 Locus coeruleus mediates cold stress-induced polycystic ovary in rats. Endocrinology 149 2907-2916. (doi:10.1210/en.20071254)

Brundu B, Loucks TL, Adler LJ, Cameron JL \& Berga SL 2006 Increased cortisol in the cerebrospinal fluid of women with functional hypothalamic amenorrhea. Journal of Clinical Endocrinology and Metabolism 91 1561-1565. (doi:10.1210/jc.2005-2422)

Dissen GA, Romero C, Paredes A \& Ojeda SR 2002 Neurotrophic control of ovarian development. Microscopy Research and Technique 59 509-515. (doi:10.1002/jemt.10227)

Dissen GA, Garcia-Rudaz C, Paredes A, Mayer C, Mayerhofer A \& Ojeda SR 2009 Excessive ovarian production of nerve growth factor facilitates development of cystic ovarian morphology in mice and is a feature of polycystic ovarian syndrome in humans. Endocrinology $\mathbf{1 5 0}$ 2906-2914. (doi:10.1210/en.2008-1575)

Dorfman M, Arancibia S, Fiedler JL \& Lara HE 2003 Chronic intermittent cold stress activates ovarian sympathetic nerves and modifies ovarian follicular development in the rat. Biology of Reproduction 68 2038-2043. (doi:10.1095/biolreprod.102.008318)

Dorfman M, Ramirez VD, Stener-Victorin E \& Lara HE 2009 Chronicintermittent cold stress in rats induces selective ovarian insulin resistance. Biology of Reproduction 80 264-271. (doi:10.1095/biol reprod.108.070904)

Ferin M 2006 Stress and the reproductive system. In Knobil and Neill's Physiology of Reproduction, 3rd edn, pp 2627-2696. Ed. JD Neill. San Diego, CA: Elsevier Academic Press.

Gerendai I, Tóth IE, Boldogkoi Z, Medveczky I \& Halász B 1998 Neuronal labeling in the rat brain and spinal cord from the ovary using viral transneuronal tracing technique. Neuroendocrinology 68 244-256. (doi:10.1159/000054372)

Greiner M, Paredes A, Araya V \& Lara HE 2005 Role of stress and sympathetic innvervation in the development of polycystic ovary syndrome. Endocrine 28 319-324. (doi:10.1385/ENDO:28:3:319)

Heider U, Pedal I \& Spanel-Borowski K 2001 Increase in nerve fibers and loss of mast cells in polycystic and postmenopausal ovaries. Fertility and Sterility 75 1141-1147. (doi:10.1016/S0015-0282(01)01805-2)

Iwasa T, Matsuzaki T, Murakami M, Kinouchi R, Shimizu F, Kuwahara A, Yasui T \& Irahara M 2009 Neonatal immune challenge affects the regulation of estrus cyclicity and feeding behaviour in female rats. International Journal of Developmental Neuroscience 27 111-114. (doi:10.1016/j.ijdevneu.2008.10.003)
Kezele P, Nilsson E \& Skinner MK 2002 Endocrinol cell-cell interactions in primordial follicles assembly and development. Frontiers in Bioscience 7 1990-1996. (doi:10.2741/kezele)

Kinoshita M, Tsukamura H, Adachi S, Matsui H, Uenoyama Y, Iwata K, Yamada S, Inoue K, Ohtaki T, Matsumoto H et al. 2005 Involvement of central metastin in the regulation of preovulatory luteinizing hormone surge and estrous cyclicity in female rats. Endocrinology $\mathbf{1 4 6}$ 4431-4436. (doi:10.1210/en.2005-0195)

Kinsey-Jones JS, Li XF, Knox AM, Wilkinson ES, Zhu XL, Chaudhary AA, Milligan SR, Lightman SL \& O'Byrne KT 2009 Down-regulation of hypothalamic kisspeptin and its receptor, kiss1r, mRNA expression is associated with stress-induced suppression of luteinising hormone secretion in the female rat. Journal of Neuroendocrinology 21 20-29. (doi:10.1111/j.1365-2826.2008.01807.x)

Knox AM, Li XF, Kinsey-Jones JS, Wilkinson ES, Wu XQ, Cheng YS, Milligan SR, Lightman SL \& O'Byrne KT 2009 Neonatal lipopolysaccharide exposure delays puberty and alters hypothalamic Kiss1 and Kiss1 r mRNA expression in the female rat. Journal of Neuroendocrinology 21 683-689. (doi:10.1111/j.1365-2826.2009.01885.x)

Lara HE, McDonald JK \& Ojeda SR 1990 Involvement of nerve growth factor in female sexual development. Endocrinology 126 364-375. (doi:10.1210/endo-126-1-364)

Lara HE, Ferruz JL, Luza S, Bustamante DA, Borges Y \& Ojeda SR 1993 Activation of ovarian sympathetic nerves in polycystic ovary syndrome. Endocrinology 133 2690-2695. (doi:10.1210/en.133.6.2690)

Lara HE, Dissen GA, Leyton V, Paredes A, Fuenzalida H, Fiedler JL \& Ojeda SR 2000 An increased intraovarian synthesis of nerve growth factor and its low affinity receptor is a principal component of steroidinduced polycystic ovary in the rat. Endocrinology 141 1059-1072. (doi:10.1210/en.141.3.1059)

Li XF, Kinsey-Jones JS, Knox AM, Wu XQ, Tahsinsoy D, Brain SD, Lightman SL \& O'Byrne KT 2007 Neonatal lipopolysaccharide exposure exacerbates stress-induced suppression of luteinizing hormone pulse frequency in adulthood. Endocrinology 148 5984-5990. (doi:10.1210/ en.2007-0710)

Li XF, Kinsey-Jones JS, Cheng Y, Knox AM, Lin Y, Petrou NA, Lightman SL, Milligan SR, Millar RP \& O'Byrne KT 2009 Kisspeptin signalling in the hypothalamic arcuate nucleus regulates $\mathrm{GnRH}$ pulse generator frequency in the rat. PLoS ONE 4 e8334. (doi:10.1371/journal.pone.0008334)

Macfarlane MS, Breen KM, Sakurai H, Adams BM \& Adams TE 2000 Effect of duration of infusion of stress-like concentration of cortisol on follicular development and the preovulatory surge of $\mathrm{LH}$ in sheep. Animal Reproduction Science 63 167-175. (doi:10.1016/S0378-4320(00)00179-2)

Mannerås L, Cajander S, Lönn M \& Stener-Victorin E 2009 Acupuncture and exercise restore adipose tissue expression of sympathetic markers and improve ovaries morphology in rats with dihydrotestosteroneinduced PCOS. American Journal of Physiology. Regulatory, Integrative and Comparative Physiology 296 R1124-R1131. (doi:10.1152/ajpregu. 90947.2008)

Marcondes FK, Bianchi FJ \& Tanno AP 2002 Determination of the estrous cycle phases of rats: some helpful considerations. Brazilian Journal of Biology 62 609-614. (doi:10.1590/S1519-69842002000400008)

Navarro VM, Castellano JM, García-Galiano D \& Tena-Sempere M 2007 Neuroendocrine factors in the initiation of puberty: the emergent role of kisspeptin. Reviews in Endocrine and Metabolic Disorders 8 11-20. (doi:10.1007/s11154-007-9028-2)

Nillson C, Larsson BM, Jennische E, Eriksson E, Björntorp P, York DA \& Holmäng A 2001 Maternal endotoxemia results in obesity and insulin resistance in adult male offspring. Endocrinology 142 2622-2630. (doi:10.1210/en.142.6.2622)

Nillson C, Jennische E, Ho HP, Eriksson E, Björntorp P \& Holmäng A 2002 Postnatal endotoxin exposure results in increase insulin sensitivity and altered activity of neuroendocrine axes in adult female rats. European Journal of Endocrinology 146 251-260. (doi:10.1530/eje.0.1460251)

Ojeda SR \& Lara HE 1989 Role of the sympathetic nervous system in the regulation of ovarian function. In The Menstrual Cycle and Its Disorders, pp 26-32. Eds KM Prike, W Wuttke \& U Scheiverg. Berlin: SpringerVerlag.

Paredes A, Gálvez A, Leyton V, Aravena G, Fiedler JL, Bustamante D \& Lara HE 1998 Stress promotes development of ovarian cysts in rats: the possible role of sympathetic nerve activation. Endocrine 8 309-315. (doi:10.1385/ENDO:8:3:309) 
Peter AT, Bosu WT \& DeDecker RJ 1989 Suppression of preovulatory luteinizing hormone surges in heifers after intrauterine infusions of Escherichia coli endotoxin. American Journal of Veterinary Research $\mathbf{5 0}$ 368-373.

Pineda R, Garcia-Galiano D, Roseweir A, Romero M, Sanchez-Garrido MA, Ruiz-Pino F, Morgan K, Pinilla L, Millar RP \& Tena-Sempere M 2010 Critical roles of kisspeptins in female puberty and preovulatory gonadotropin surges as revealed by novel antagonist. Endocrinology 151 722-730. (doi:10.1210/en.2009-0803)

Rajah R, Glaser EM \& Hirshfield AN 1992 The changing architecture of the neonatal rat ovary during histogenesis. Developmental Dynamics 194 177-192. (doi:10.1002/aja.1001940303)

Rivest S, Lee S, Attardi B \& Rivier C 1993 The chronic intracerebroventricular infusion of interleukin- $1 \beta$ alters the activity of the hypothalamicpituitary-gonadal axis of cycling rats. I. Effect on LHRH and gonadotropin biosynthesis and secretion. Endocrinology 133 2424-2430. (doi:10.1210/ en.133.6.2424)

Roozendaal MM, Swarts JJ, van Maanen JC, Wiegant VM \& Mattheij JA 1997 Inhibition of the LH surge in cyclic rats by stress is not mediated by opioids. Life Sciences 60 735-742. (doi:10.1016/S0024-3205(96) 00652-2)

Rosa-E-Silva A, Guimaraes MA, Padmanabhan V \& Lara HE 2003 Prepubertal administration of estradiol valerate disrupts cyclicity and leads to cystic ovarian morphology during adult life in the rat: role of sympathetic innervation. Endocrinology 144 4289-4297. (doi:10.1210/ en.2003-0146)

Shanks N, Larocque S \& Meaney MJ 1995 Neonatal endotoxin exposure alters the development of the hypothalamic-pituitary-adrenal axis: early illness and later responsivity to stress. Journal of Neuroscience $\mathbf{1 5}$ 376-384.

Shanks N, Windle RJ, Perks PA, Haubuz MS, Jessop DS, Ingram CD \& Lightman SL 2000 Early-life exposure to endotoxin alters hypothalamicpituitary-adrenal function and predisposition to inflammation. PNAS 97 5645-5650. (doi:10.1073/pnas.090571897)

Sotomayor-Zarate R, Dorfman M, Paredes A \& Lara HE 2008 Neonatal exposure to estradiol valerate programs ovarian sympathetic innervation and follicular development in the adult rat. Biology of Reproduction $\mathbf{7 8}$ 673-680. (doi:10.1095/biolreprod.107.063974)
Suh BY, Liu JH, Berga SL, Quigley ME, Laughlin GA \& Yen SS 1988 Hypercortisolism in patients with functional hypothalamic-amenorrhea. Journal of Clinical Endocrinology and Metabolism 66 733-739. (doi:10. 1210/jcem-66-4-733)

Sverrisdóttir YB, Mogren T, Kataoka J, Janson PO \& Stener-Victorin E 2008 Is polycystic ovary syndrome associated with high sympathetic nerve activity and size at birth? American Journal of Physiology. Endocrinology and Metabolism 294 E576-E581. (doi:10.1152/ajpendo.00725.2007)

Turner BB \& Taylor AN 1977 Effects of postnatal corticosterone treatment on reproductive development in the rat. Journal of Reproduction and Fertility 51 309-314. (doi:10.1530/jrf.0.0510309)

Valli G, Sudha S, Ravi Sankar B, Govindarajulu P \& Srinivasan N 2000 Altered corticosterone status impairs steroidogenesis in the granulosa and thecal cells of Wistar rats. Journal of Steroid Biochemistry and Molecular Biology 73 153-158. (doi:10.1016/S0960-0760(00)00063-7)

Wagenmaker ER, Breen KM, Oakley AE, Pierce BN, Tilbrook AJ, Turner AI \& Karsch FJ 2009 Cortisol interferes with the estradiol-induced surge of luteinizing hormone in the ewe. Biology of Reproduction 80 458-463. (doi:10.1095/biolreprod.108.074252)

Xia Y \& Krukoff TL 2003 Differential neuronal activation in the hypothalamic paraventricular nucleus and autonomic/neuroendocrine responses to I.C.V. endotoxin. Neuroscience 121 219-231. (doi:10. 1016/S0306-4522(03)00290-2)

Xiao E, Xia-Zhang L, Barth A, Zhu J \& Ferin M 1998 Stress and the menstrual cycle: relevance of cycle quality in the short- and long-term response to a 5-day endotoxin challenge during the follicular phase in the rhesus monkey. Journal of Clinical Endocrinology and Metabolism 83 2454-2460. (doi:10.1210/jc.83.7.2454)

Zhou ZZ \& Jones SB 1993 Involvement of central vs. peripheral mechanisms in mediating sympathoadrenal activation in endotoxic rats. American Journal of Physiology 265 R683-R688.

Received 25 May 2010

First decision 25 June 2010

Revised manuscript received 1 November 2010

Accepted 17 November 2010 